\title{
Effectiveness of Amlodipine on Blood Pressure Control in Hypertensive Patients in India: A Real-World, Retrospective Study from Electronic Medical Records
}

\author{
Mohammed Yunus Khan ${ }^{1}$ (D) . Sucheta Pandit ${ }^{1} \cdot$ Saumitra Ray $^{2} \cdot$ Jagdish Chander Mohan $^{3} \cdot$ B. C. Srinivas ${ }^{4}$. \\ Santosh Ramakrishnan ${ }^{5}$. Amey Mane ${ }^{1}$. Suyog Mehta ${ }^{1}$. Snehal Shah ${ }^{6}$
}

Published online: 8 September 2020

(c) The Author(s) 2020

\begin{abstract}
Background The effectiveness of amlodipine has been reported in clinical trials in India. However, real-world data on the effectiveness of amlodipine in India is limited.

Objective To provide real-world evidence regarding the effectiveness of amlodipine as monotherapy or in combination with other antihypertensive drugs (AHDs) in Indian patients with essential hypertension.

Methods Electronic medical record data of adult patients who were diagnosed with essential hypertension $(\geq 140 / 90 \mathrm{mmHg})$ and were prescribed amlodipine as monotherapy or add-on therapy were retrospectively analyzed. Patients were classified based on the number of AHD classes prescribed on initiation of amlodipine. Change in systolic (SBP) and diastolic (DBP) blood pressure from baseline was the primary endpoint. Evaluation of proportion of patients who achieved treatment goals as per 2018 European Society of Cardiology/European Society of Hypertension guidelines was the secondary endpoint. Readings were obtained before initiating amlodipine and after at least a month of therapy with amlodipine.

Results Among the 462 included patients, the majority (90.7\%) were on amlodipine monotherapy or amlodipine +1 AHD. Mean (95\% confidence interval [CI]) change in the amlodipine monotherapy group was: SBP $(-12.1[-14.9,-9.3] \mathrm{mmHg})$ and DBP $(-7.5[-8.9,-6.1] \mathrm{mmHg})$ and mean $(95 \% \mathrm{CI})$ change in the amlodipine +1 AHD group was: SBP $(-17.8$ $[-21.0,-14.6] \mathrm{mmHg})$ and DBP $(-9.5[-11.0,-8.0] \mathrm{mmHg})(P<0.001$ for all $)$. SBP and DBP goals were achieved by $31.4 \%$ and $42.9 \%$ of patients on amlodipine monotherapy and by $38.9 \%$ and $51.8 \%$ of patients on amlodipine $+1 \mathrm{AHD}$, respectively. Among patients aged $\leq 45$ years, mean $(95 \% \mathrm{CI})$ change in the amlodipine monotherapy group was: SBP $(-11.7[-16.0,-7.4] \mathrm{mmHg} ; P<0.001)$ and DBP $(-7.2[-9.7,-4.7] \mathrm{mmHg} ; P<0.001)$ and mean $(95 \% \mathrm{CI}) \mathrm{change}$ in the amlodipine + 1AHD group was: SBP $(-14.6[-21.9,-7.3] \mathrm{mmHg} ; P<0.05)$ and DBP $(-10.6[-14.8,-6.4]$ $\mathrm{mmHg} ; P<0.01)$. SBP and DBP goals were achieved by $35.4 \%$ and $33.8 \%$ of patients on amlodipine monotherapy and by $48.0 \%$ and $56.0 \%$ of patients on amlodipine +1 AHD, respectively. Among patients aged $\geq 65$ years, mean (95\% CI) change in the amlodipine monotherapy group was: SBP $(-13.9[-20.2,-7.6] \mathrm{mmHg} ; P<0.01)$ and DBP $(-8.5[-11.4,-5.7]$ $\mathrm{mmHg} ; P<0.001)$ and mean $(95 \% \mathrm{CI})$ change in the amlodipine $+1 \mathrm{AHD}$ group was: SBP $(-22.4[--28.8,-16.0] \mathrm{mmHg}$; $P<0.001)$ and DBP $(-10.8[-14.0,-7.6] \mathrm{mmHg} ; P<0.001)$. SBP and DBP goals were achieved by $25.5 \%$ and $13.7 \%$ of patients on amlodipine monotherapy and by $29.8 \%$ and $14.0 \%$ of patients on amlodipine +1 AHD.

Conclusion Amlodipine prescribed as monotherapy or add-on therapy during routine clinical practice significantly reduced $\mathrm{BP}$ in $\leq 45$ - and $\geq 65$-year-old Indian patients with mild to moderate hypertension, emphasizing that amlodipine may be a good candidate for BP control in Indian patients with essential hypertension in these age groups.
\end{abstract}

Mohammed Yunus Khan

myunuskhan@drreddys.com

Extended author information available on the last page of the article 


\section{Key Points}

We evaluated the effectiveness of amlodipine prescribed to Indian patients with hypertension during routine clinical practice in India.

Amlodipine was found to be effective in reducing blood pressure in patients aged $\leq 45$ years as well as $\geq 65$ years during routine clinical practice in India.

\section{Introduction}

The prevalence of hypertension has been increasing worldwide [1], with around 234 million hypertensive adults reported in India itself [2]. Hypertension-associated morbidity and mortality in India is also high. The Global Burden of Disease 2017 study reported that hypertensive heart disease caused around 2.5 million disability-adjusted life-years and 98,912 deaths in India [3]. Despite the increased prevalence and negative outcomes attributed to hypertension [4], the awareness, treatment, and control of hypertension in India remains poor [5, 6].

Many guidelines such as the 2014 Eighth Joint National Committee evidence-based guidelines [7], 2017 American College of Cardiology guidelines [8], 2018 European Society of Cardiology guidelines [9], and 2019 Indian Guidelines on Hypertension-IV [10] describe the definition, evaluation, classification, and management of hypertension. Calcium channel blockers (CCBs) are some of the first-line agents recommended by these guidelines to combat essential hypertension. Consistent with these guidelines, multiple studies have reported the prescription of CCBs in India, either as monotherapy or in combination with other antihypertensive drugs (AHDs), to treat hypertension [11-16]. Moreover, among the CCBs, amlodipine was preferred by $75.7 \%$ of Indian physicians [11].

The efficacy of amlodipine in Indian patients with hypertension has been reported by many clinical trials [17-21]. However, real-world electronic medical record (EMR) evidence regarding the effectiveness of amlodipine in India is unavailable. Therefore, we conducted this study to provide evidence regarding the effectiveness of amlodipine as monotherapy or add-on therapy among Indian patients with hypertension in a real-world setting.

\section{Methods}

\subsection{Data Source(s)}

Analysis was performed from an Indian electronic software owned and administered by HealthPlix Technologies PRV. This software has been in operation since 2016 and fulfils the day-to-day operational needs of 12 medical specialties across $150+$ cities in 20 states. Information including demographics, diagnoses, medications, cardiac risk factors, tests and procedures conducted, functional status, and other data elements obtained from the software were used to conduct the analysis.

Applicable national regulatory laws and guidelines were followed while conducting the study. The study protocol was approved by an independent ethics committee on 3 December 2019. Patient confidentiality was maintained at all times as the study was performed using anonymized information only.

\subsection{Study Design and Sample Selection}

In this retrospective observational study, HealthPlix Technologies PRV assessed electronic medical records (EMR) data of Indian patients diagnosed with essential hypertension, and mapped the brand name on the prescriptions with the generic name to identify the prescribed AHDs. From January 2018 to September 2019, adult patients ( $\geq 18$ years old) who were diagnosed with essential hypertension by their physicians as per ESC/ESH 2018 guidelines ( $\geq 140 / 90 \mathrm{mmHg}$ ) at baseline, were prescribed amlodipine either as monotherapy or as add-on therapy, and had data available for at least two visits with a minimum gap of 1 month after initiation of amlodipine, were included in the study. Patients diagnosed with secondary hypertension and those on other CCBs at visit 1 (baseline) were excluded from the study.

Each patient was required to have at least two valid blood pressure (BP) readings in the EMR. The BP measurement taken on the day of amlodipine initiation was considered as the Visit 1 (baseline) reading and the first available BP reading after at least $\geq 30$ days of amlodipine initiation was considered as the Visit 2 reading. The $\geq 30$-day gap was observed as a smaller gap between visits might result in inaccurate analyses. This reading was required to be obtained before discontinuation of amlodipine, initiation of any other AHD, or end of study period, whichever occurred first. Any further readings available till amlodipine discontinuation, addition of new therapy, or up to end of study were collected. 


\subsection{Study Endpoints}

Evaluation of mean change in systolic and diastolic BP (SBP and DBP) from baseline to post-index (amlodipine discontinuation, addition of new therapy, or up to end of study) in patients prescribed amlodipine was the primary endpoint. Assessment of the proportion of patients with essential hypertension on amlodipine who achieved individualized BP goals (SBP and DBP) as per ESC/ESH 2018 guidelines from baseline to post-index (amlodipine discontinuation, addition of new therapy, or up to end of study) was the secondary endpoint. Subgroup analysis (age, gender, diabetes, chronic kidney disease [CKD], coronary artery disease, stroke, dyslipidemia) was also performed for these endpoints.

\subsection{Assessments}

Data regarding demographic characteristics such as age, gender, personal and family history, and clinical characteristics such as grade of hypertension as per ESC/ESH 2018 guidelines, BP readings, co-morbidities, medications used within the previous 30 days, and laboratory data and electrocardiogram in the last 6 months (if available) were collected at baseline. Data regarding BP, medications in use, and laboratory data were collected from the next visit.

Prior antihypertensive therapy was defined as the use of any AHDs before initiation of amlodipine that were not discontinued on or before starting treatment with amlodipine. Prior AHDs were classified into: angiotensin-converting enzyme inhibitors/angiotensin II receptor blockers, $\beta$-blockers, diuretics, and others ( $\alpha$-blockers, vasodilators, methyldopa, clonidine, and mineralocorticoid receptor antagonists). Patients on fixed-dose combination products were classified as receiving AHDs in each class. Additionally, patients were classified based on the number of AHD classes they received while initiating amlodipine therapy into: no other AHD (amlodipine monotherapy), amlodipine + 1AHD, amlodipine + 2AHDs, and amlodipine + 3AHDs.

\subsection{Statistical Analysis}

Descriptive statistics were used to summarize the study variables. Frequency and percentages were reported for categorical variables while means and standard deviations were reported for continuous variables. Changes in continuous variables were reported as mean change with $95 \%$ confidence intervals (CIs). $P$ values were calculated using the Altman and Bland method [22] and $P<0.05$ was considered statistically significant.

\section{Results}

\subsection{Baseline Characteristics}

Among the patients who met the inclusion criteria and were included in the study $(N=462$, Fig. 1$)$, the majority $(90.7 \%)$ were prescribed amlodipine monotherapy or amlodipine +1 AHD. Patients on amlodipine monotherapy were the youngest (mean \pm SD age $54.1 \pm 13.6$ years), while patients on amlodipine +2 AHDs were the eldest (mean \pm SD age $62.5 \pm 14.6$ years). The highest proportion of females was present in the amlodipine +1 AHD group $(56.5 \%)$. Patients on amlodipine monotherapy reported the highest mean weight $(73.0 \mathrm{~kg})$. The highest proportion of diabetes mellitus (55.6\%) and cardiovascular events $(38.5 \%)$ was reported by patients on amlodipine + 2AHDs. Patients on amlodipine + 3AHDs reported the highest proportion of CKD (4.2\%). The majority of the patients on amlodipine monotherapy were treatmentnaïve $(73.5 \%)$. All the patients in the other groups were treatment-experienced users. Grade 1 hypertension was more common in patients on amlodipine monotherapy but grade 2 SBP and grade 1 DBP was more common than other grades in patients on amlodipine along with other AHDs. The majority of the patients in each group were prescribed a $5 \mathrm{mg}$ dose of amlodipine and most of the patients in all the groups were diagnosed and treated by a consulting physician or cardiologist (Table 1). The average duration between visits 1 and 2 was 91.2 days, 100.9 days, 78.8 days, and 51.2 days for patients on amlodipine monotherapy, amlodipine + 1AHD, amlodipine + 2AHDs, and amlodipine +3AHDs, respectively.

\subsection{Primary Endpoint}

Statistically significant reductions in SBP and DBP $(P<0.001$ for all $)$ were observed in patients on amlodipine monotherapy and amlodipine +1 AHD (Table 2). The greatest mean $(95 \% \mathrm{CI})$ change in SBP $(-17.8[-21.0$, - 14.6] $\mathrm{mmHg} ; P<0.001)$ and DBP $(-9.5[-11.0,-8.0]$ $\mathrm{mmHg} ; P<0.001)$ was observed in patients on amlodipine +1 AHD (Fig. 2).

Table 3 contains the subgroup analysis results as per age. Patients were classified according to age into three subgroups: $\leq 45$ years, $<65$ years, and $\geq 65$ years. In the $\leq 45$ years subgroup, the greatest mean $(95 \% \mathrm{CI})$ change in SBP $(-27.1[-39.5,-14.7] \mathrm{mmHg} ; P<0.05)$ was observed in patients on amlodipine +2AHDs and the greatest mean $(95 \% \mathrm{CI})$ change in DBP $(-10.6[-14.8$, - 6.4] $\mathrm{mmHg} ; P<0.01)$ was observed in patients on amlodipine +1 AHD. In the $<65$ years subgroup, the 


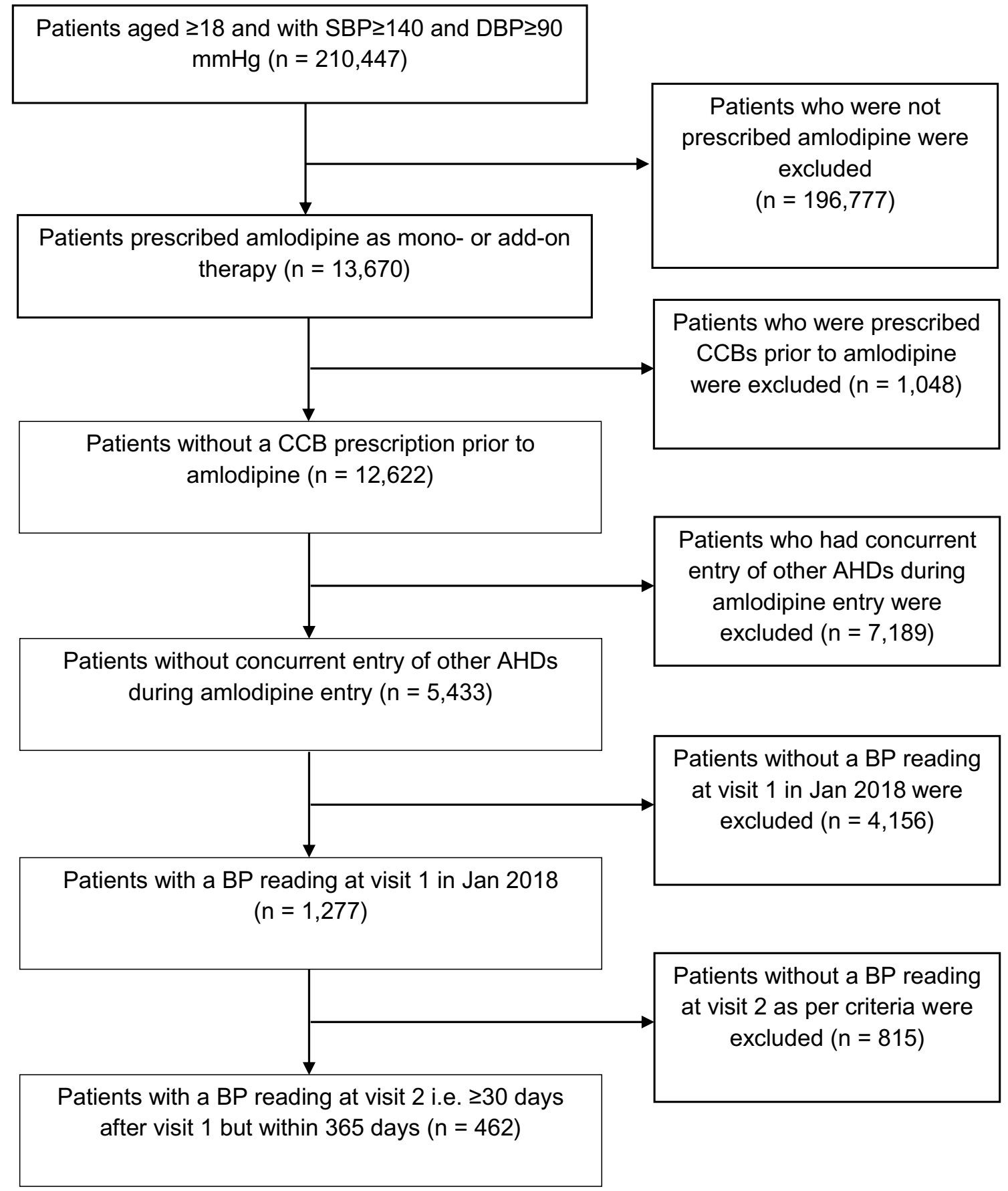

Fig. 1 Patient flowchart. $A H D$ antihypertensive drug, $C C B$ calcium channel blocker, $D B P$ diastolic blood pressure, $S B P$ systolic blood pressure, $H T$ antihypertensive

greatest mean $(95 \% \mathrm{CI})$ change in SBP $(-16.0[-19.5$, - 12.5] $\mathrm{mmHg} ; P<0.01)$ was observed in patients on amlodipine $+1 \mathrm{AHD}$ and greatest statistically significant mean $(95 \% \mathrm{CI})$ change in DBP $(-9.0[-10.7,-7.3]$ $\mathrm{mmHg} ; P<0.001)$ was observed in patients on amlodipine +1 AHD. In the $\geq 65$ years subgroup, patients on amlodipine +1 AHD reported both the greatest mean
(95\% CI) change in SBP $(-22.4[-28.8,-16.0] \mathrm{mmHg}$; $P<0.001)$ and DBP $(-10.8[-14.0,-7.6] \mathrm{mmHg}$; $P<0.001)$.

Among patients with essential hypertension and comorbid diabetes, patients on amlodipine + 1AHD showed the greatest statistically significant mean $(95 \% \mathrm{CI})$ change in SBP $(-17.8[-22.5,-13.1] \mathrm{mmHg} ; P<0.001)$ and 
Table 1 Demographic and clinical characteristics at baseline

\begin{tabular}{|c|c|c|c|c|}
\hline Parameter & $\begin{array}{l}\text { Amlodipine } \\
\text { monotherapy } \\
(n=226)\end{array}$ & $\begin{array}{l}\text { Amlodipine } \\
+1 \mathrm{AHD} \\
(n=193)\end{array}$ & $\begin{array}{l}\text { Amlodipine } \\
+2 \text { AHDs } \\
(n=36)\end{array}$ & $\begin{array}{l}\text { Amlodipine } \\
+3 \text { AHDs } \\
(n=7)\end{array}$ \\
\hline Age (years, mean [SD]) & $54.1(13.6)$ & $58.0(12.6)$ & $62.5(14.6)$ & $55.7(7.1)$ \\
\hline Females $(n[\%[)$ & $115(50.6)$ & $109(56.5)$ & $16(44.4)$ & $2(28.6)$ \\
\hline Weight $(\mathrm{kg} \text {, mean }[\mathrm{SD}])^{\mathrm{a}}$ & $73.0(53.2)$ & $71.4(15.5)$ & $66.9(8.3)$ & $70.9(4.4)$ \\
\hline \multicolumn{5}{|l|}{ Co-morbidities $(n[\%])$} \\
\hline Diabetes mellitus & $67(29.6)$ & $83(43.0)$ & $20(55.6)$ & $3(42.9)$ \\
\hline $\mathrm{CV}$ events ${ }^{\mathrm{b}}$ & $16(11.6)$ & $27(20.6)$ & $10(38.5)$ & $2(33.3)$ \\
\hline $\mathrm{CKD}^{\mathrm{b}}$ & $3(2.2)$ & $2(1.5)$ & $1(3.8)$ & $1(4.2)$ \\
\hline \multicolumn{5}{|l|}{ Patient type ( $n[\%])$} \\
\hline Treatment-naïve & $166(73.5)$ & 0 & 0 & 0 \\
\hline Treatment-experienced & $60(36.1)$ & $193(100)$ & $36(100)$ & $7(100)$ \\
\hline \multicolumn{5}{|l|}{ Hypertension grade ( $n[\%])$} \\
\hline Grade 1 SBP & $101(44.7)$ & $74(38.3)$ & $11(30.6)$ & $2(28.6)$ \\
\hline Grade $1 \mathrm{DBP}$ & $120(53.1)$ & $112(58.0)$ & $19(52.8)$ & $3(42.9)$ \\
\hline Grade 2 SBP & $93(41.2)$ & $82(42.5)$ & $17(47.2)$ & $3(42.9)$ \\
\hline Grade 2 DBP & $87(38.5)$ & $64(33.2)$ & $14(38.9)$ & $4(57.1)$ \\
\hline Grade 3 SBP & $32(14.2)$ & $37(19.2)$ & $8(22.2)$ & $2(28.6)$ \\
\hline Grade 3 DBP & $19(8.4)$ & $17(8.8)$ & $3(8.3)$ & $0(0.0)$ \\
\hline \multicolumn{5}{|c|}{ Amlodipine dose strength $(n[\%])$} \\
\hline $2.5 \mathrm{mg}$ & $56(24.8)$ & $51(26.4)$ & $7(19.4)$ & 0 \\
\hline $5 \mathrm{mg}$ & $160(70.8)$ & $139(72.0)$ & $27(75.0)$ & $7(100)$ \\
\hline $10 \mathrm{mg}$ & $10(4.4)$ & $3(1.6)$ & $2(5.6)$ & 0 \\
\hline \multicolumn{5}{|l|}{ Physician type ( $n[\%])$} \\
\hline Consulting physician & $118(52.2)$ & $70(36.3)$ & $13(36.1)$ & $2(28.6)$ \\
\hline Cardiologist & $54(23.9)$ & $78(40.4)$ & $15(41.7)$ & $2(28.6)$ \\
\hline Diabetologist & $20(8.8)$ & $24(12.4)$ & $8(22.2)$ & $1(14.3)$ \\
\hline Endocrinologist & $20(8.8)$ & $20(10.4)$ & 0 & $1(14.3)$ \\
\hline General physician & $10(4.4)$ & $1(0.5)$ & 0 & $1(14.3)$ \\
\hline Nephrologist & $4(1.8)$ & 0 & 0 & \\
\hline
\end{tabular}

$A H D$ antihypertensive drug, $C K D$ chronic kidney disease, $C V$ cardiovascular, $D B P$ diastolic blood pressure, $S B P$ systolic blood pressure, $S D$ standard deviation

${ }^{a}$ The sample sizes for weight assessment were: Amlodipine monotherapy $(n=101)$, Amlodipine +1 AHD $(n=93)$, Amlodipine +2 AHDs $(n=15)$, and Amlodipine +3 AHDs $(n=3)$

${ }^{\mathrm{b}}$ The sample sizes for assessment of number of patients with CV events and CKD is different from the sample size of the group as a limited number of patients had the diagnosis mentioned in the diagnosis field. Hence, the sample sizes were: Amlodipine monotherapy $(n=137)$, Amlodipine +1AHD $(n=131)$, Amlodipine +2 AHDs $(n=26)$, and Amlodipine +3 AHDs $(n=6)$. CV events comprise coronary artery disease, myocardial infarction, and stroke
DBP $(-10.0[-12.5,-7.5] \mathrm{mmHg} ; P<0.001)$. Among patients with essential hypertension and co-morbid dyslipidemia, patients on amlodipine +2AHDs showed the greatest mean $(95 \% \mathrm{CI})$ change in SBP $(-24.7[-37.0$, $-12.4] \mathrm{mmHg} ; P<0.05)$ and patients on amlodipine monotherapy showed the greatest mean $(95 \% \mathrm{CI})$ change in DBP $(-9.3[-12.2,-6.4] \mathrm{mmHg} ; P<0.001)$ (Table 4). Among the seven patients with essential hypertension and CKD, amlodipine initiation reduced mean (SD) SBP from 160.0 (10.7) to $151.7(16.3) \mathrm{mmHg}$ (mean [95\% CI] change in SBP - $8.3[-21.5,4.9] \mathrm{mmHg}$ ) and mean (SD) DBP from 96.3 (10.3) to 87.1 (14.0) $\mathrm{mmHg}$ (mean [95\%
CI] change in DBP - $9.1[-14.5,-3.7] \mathrm{mmHg}$ ). Data of patients with co-morbid coronary artery disease and stroke were minimal and hence were not presented.

Among male patients with essential hypertension, the greatest mean $(95 \% \mathrm{CI})$ change in both SBP $(-18.1$ $[-22.6,-13.6] \mathrm{mmHg} ; P<0.001)$ and DBP $(-9.6$ $[-11.6,-7.6] \mathrm{mmHg} ; P<0.001)$ was observed in patients on amlodipine +1 AHD. Similarly, among female patients with essential hypertension, the greatest statistically significant mean $(95 \% \mathrm{CI})$ change in both SBP $(-17.6[-22.0,-13.2] \mathrm{mmHg} ; P<0.001)$ and DBP 
Table 2 Effect of amlodipine on blood pressure

\begin{tabular}{lllll}
\hline Parameter & $\begin{array}{l}\text { Amlodipine } \\
\text { monotherapy } \\
(n=226)\end{array}$ & $\begin{array}{l}\text { Amlodipine } \\
+1 \mathrm{AHD} \\
(n=193)\end{array}$ & $\begin{array}{l}\text { Amlodipine } \\
+2 \text { AHDs }(n=36)\end{array}$ & $\begin{array}{l}\text { Amlodipine } \\
+3 \text { AHDs } \\
(n=7)\end{array}$ \\
\hline $\begin{array}{l}\text { SBP (mmHg, mean [SD]) } \\
\text { Visit } 1\end{array}$ & $160.0(14.7)$ & $162.5(16.0)$ & $165.6(20.0)$ & $165.3(19.4)$ \\
Visit 2 & $147.8(20.0)$ & $144.7(19.9)$ & $151.9(27.0)$ & $154.3(15.9)$ \\
$P$ value & $<0.001$ & $<0.001$ & 0.401 & 1.08 \\
DBP (mmHg, mean [SD]) & & & $96.4(7.8)$ & $96.9(5.1)$ \\
Visit 1 & $96.3(6.6)$ & $96.0(7.4)$ & $89.6(10.4)$ & $88.6(6.4)$ \\
Visit 2 & $88.8(10.4)$ & $86.5(10.2)$ & 0.148 & 0.322 \\
$P$ value & $<0.001$ & $<0.001$ & & \\
\hline
\end{tabular}

Visit 2 readings were statistically compared against Visit 1 readings

$A H D$ antihypertensive drug, $D B P$ diastolic blood pressure, $S B P$ systolic blood pressure, $S D$ standard deviation

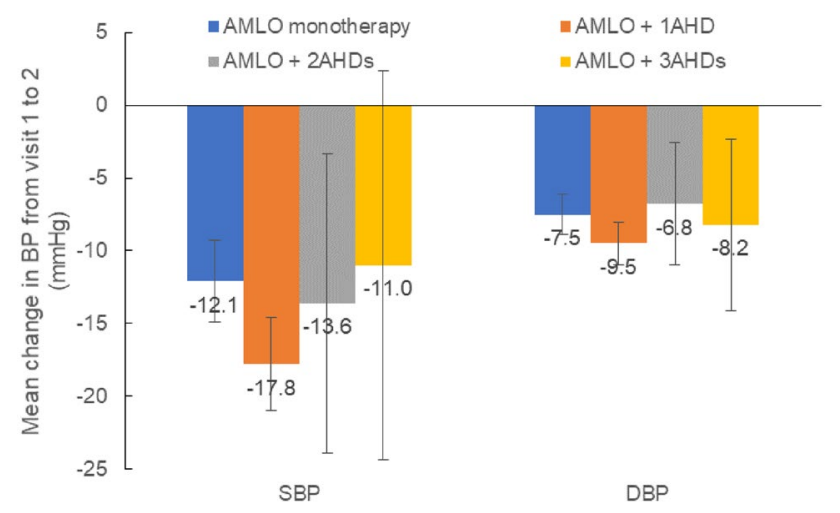

Fig. 2 Amlodipine-induced change in blood pressure. Mean (95\% confidence interval) change in blood pressure from visit 1 to visit 2 is shown. Visit 2 readings were statistically compared against Visit 1 readings. ${ }^{* * *} P<0.001$. AHD antihypertensive drug, $A M L O$ amlodipine, $B P$ blood pressure, $D B P$ diastolic $\mathrm{BP}, S B P$ systolic BP

$(-9.4[-11.6,-7.2] \mathrm{mmHg} ; P<0.001)$ was observed in patients on amlodipine +1 AHD (Table 4).

\subsection{Secondary Endpoint}

Overall, $34.4 \%$ of the patients achieved SBP and $46.5 \%$ achieved DBP goal as per the ESC/ESH 2018 guidelines. Group-wise, the highest proportion of patients who achieved their SBP goal (38.9\%) and DBP goal (51.8\%) were on amlodipine + 1AHD (Fig. 3).

Figure 4 shows age-wise subgroup analysis of the proportion of patients who achieved their BP goals as per ESC/ ESH 2018 guidelines. In patients aged $\leq 45$ years, the highest proportion of who achieved their SBP goal $(57.1 \%)$ and DBP goal $(57.1 \%)$ were on amlodipine + 2AHDs. In patients aged $\geq 65$ years, the highest proportion of patients achieving their SBP goal (29.8\%) and DBP goal (14.0\%) were on amlodipine + 1AHD.
Among patients with co-morbid diabetes, $30.1 \%$ of the patients achieved SBP goal and $42.2 \%$ of the patients achieved DBP goal as per the ESC/ESH 2018 guidelines. Group-wise, patients on amlodipine + 2AHDs achieved SBP goal the most (40.0\%) and patients on amlodipine + 1 AHD achieved DBP goal the most (45.8\%) (Fig. 5). Among patients with co-morbid dyslipidemia, $35.8 \%$ of the patients achieved SBP goal and $50.5 \%$ of the patients achieved DBP goal. Group-wise, patients on amlodipine + 1AHD achieved SBP goal the most (37.6\%) and patients on amlodipine monotherapy achieved DBP goal the most (54.0\%) (Fig. 6). Among patients with co-morbid CKD, $14.3 \%$ of the patients achieved SBP goal $(<140 \mathrm{mmHg})$ and $71.4 \%$ of the patients achieved DBP goal $(<90 \mathrm{mmHg})$ as per the ESC/ESH 2018 guidelines. Data of patients with co-morbid coronary artery disease and stroke were minimal and hence were not presented.

\section{Discussion}

Amlodipine is a second-generation CCB with high vascular selectivity that reduces peripheral resistance while preserving myocardial contractility [23]. Furthermore, amlodipine has a long elimination half-life and binds to the target receptors in a slow and sustained manner, resulting in a smooth onset of action and 24-h BP control [23]. Peripheral edema is a common adverse effect of amlodipine apart from dizziness, fatigue, headache, palpitations, and nausea [24]. These adverse effects have also been reported in real-world studies [25-27]. In addition to a favorable adverse effect profile, amlodipine has also been reported to reduce the risk of cardiovascular events and all-cause mortality compared with non-CCB AHDs [28].

The current study is one of the earliest studies in India to assess the impact of an AHD on patients with essential hypertension using EMR data. It provides new evidence 
Table 3 Effect of amlodipine on blood pressure - subgroup analysis according to age

\begin{tabular}{|c|c|c|c|c|}
\hline $\begin{array}{l}\text { Patients with hypertension } \\
\text { aged } \leq 45 \text { years }\end{array}$ & $\begin{array}{l}\text { Amlodipine monotherapy } \\
(n=65)\end{array}$ & $\begin{array}{l}\text { Amlodipine + 1AHD } \\
(n=25)\end{array}$ & $\begin{array}{l}\text { Amlodipine + 2AHDs } \\
(n=7)\end{array}$ & $\begin{array}{l}\text { Amlodipine + 3AHDs } \\
(n=1)\end{array}$ \\
\hline \multicolumn{5}{|l|}{$\mathrm{SBP}(\mathrm{mmHg}$, mean $[\mathrm{SD}])$} \\
\hline Visit 1 & $155.0(13.2)$ & $155.7(11.1)$ & $161.4(18.1)$ & $160.0(-)$ \\
\hline Visit 2 & $143.3(18.0)$ & $141.1(18.9)$ & $134.3(17.6)$ & $170.0(-)$ \\
\hline $\begin{array}{l}\text { Change from Visit } 1 \text { (mean } \\
{[95 \% \mathrm{CI}] \text { ) }}\end{array}$ & $-11.7(-16.0,-7.4)$ & $-14.6(-21.9,-7.3)$ & $-27.1(-39.5,-14.7)$ & $-10.0(-)$ \\
\hline$P$ value & $<0.001$ & 0.028 & 0.010 & - \\
\hline \multicolumn{5}{|l|}{$\mathrm{DBP}(\mathrm{mmHg}$, mean $[\mathrm{SD}])$} \\
\hline Visit 1 & $98.5(6.2)$ & $98.4(7.4)$ & $97.1(7.0)$ & $100.0(-)$ \\
\hline Visit 2 & $91.3(10.5)$ & $87.9(11.7)$ & $87.7(12.3)$ & $90.0(-)$ \\
\hline $\begin{array}{l}\text { Change from Visit } 1 \text { (mean } \\
{[95 \% \mathrm{CI}] \text { ) }}\end{array}$ & $-7.2(-9.7,-4.7)$ & $-10.6(-14.8,-6.4)$ & $-9.4(-15.6,-3.2)$ & $-10.0(-)$ \\
\hline$P$ value & $<0.001$ & 0.001 & 0.214 & - \\
\hline $\begin{array}{l}\text { Patients with hypertension } \\
\text { aged }<65 \text { years }\end{array}$ & $\begin{array}{l}\text { Amlodipine monotherapy } \\
(n=175)\end{array}$ & $\begin{array}{l}\text { Amlodipine + 1AHD } \\
(n=136)\end{array}$ & $\begin{array}{l}\text { Amlodipine + 2AHDs } \\
(n=20)\end{array}$ & $\begin{array}{l}\text { Amlodipine + 3AHDs } \\
(n=6)\end{array}$ \\
\hline \multicolumn{5}{|l|}{$\mathrm{SBP}(\mathrm{mmHg}$, mean $[\mathrm{SD}])$} \\
\hline Visit 1 & $159.3(14.9)$ & $159.4(13.3)$ & $161.6(13.5)$ & $166.2(20.8)$ \\
\hline Visit 2 & $147.6(19.8)$ & $143.4(19.8)$ & $152.3(30.1)$ & $153.3(17.0)$ \\
\hline $\begin{array}{l}\text { Change from Visit } 1 \text { (mean } \\
{[95 \% \mathrm{CI}] \text { ) }}\end{array}$ & $-11.6(-14.7,-8.6)$ & $-16.0(-19.5,-12.5)$ & $-9.3(-24.4,5.8)$ & $-12.8(-28.0,2.4)$ \\
\hline$P$ value & $<0.001$ & $<0.001$ & 1.0 & 1.0 \\
\hline \multicolumn{5}{|l|}{ DBP $(\mathrm{mmHg}$, mean [SD]) } \\
\hline Visit 1 & $97.2(6.8)$ & $96.2(7.1)$ & $96.2(5.7)$ & $98.0(4.6)$ \\
\hline Visit 2 & $90.0(10.5)$ & $87.3(10.5)$ & $92.1(11.7)$ & $88.3(6.9)$ \\
\hline $\begin{array}{l}\text { Change from Visit } 1 \text { (mean } \\
{[95 \% \mathrm{CI}] \text { ) }}\end{array}$ & $-7.2(-8.8,-5.6)$ & $-9.0(-10.7,-7.3)$ & $-4.1(-9.5,1.3)$ & $-9.7(-16.0,-3.4)$ \\
\hline$P$ value & $<0.001$ & $<0.001$ & 1.0 & 1.0 \\
\hline $\begin{array}{l}\text { Patients with hypertension } \\
\text { aged } \geq 65 \text { years }\end{array}$ & $\begin{array}{l}\text { Amlodipine monotherapy } \\
(n=51)\end{array}$ & $\begin{array}{l}\text { Amlodipine + 1AHD } \\
(n=57)\end{array}$ & $\begin{array}{l}\text { Amlodipine + 2AHDs } \\
(n=16)\end{array}$ & $\begin{array}{l}\text { Amlodipine + 3AHDs } \\
(n=1)\end{array}$ \\
\hline \multicolumn{5}{|l|}{$\mathrm{SBP}(\mathrm{mmHg}$, mean [SD]) } \\
\hline Visit 1 & $162.4(13.7)$ & $170.1(19.1)$ & $170.6(25.1)$ & $160.0(-)$ \\
\hline Visit 2 & $148.5(20.6)$ & $147.8(19.8)$ & $151.5(22.7)$ & $160.0(-)$ \\
\hline $\begin{array}{l}\text { Change from Visit } 1 \text { (mean } \\
{[95 \% \mathrm{CI}] \text { ) }}\end{array}$ & $-13.9(-20.2,-7.6)$ & $-22.4(-28.8,-16.0)$ & $-19.1(-32.0,-6.2)$ & $0(-)$ \\
\hline$P$ value & 0.009 & $<0.001$ & 0.241 & - \\
\hline \multicolumn{5}{|l|}{$\mathrm{DBP}(\mathrm{mmHg}$, mean $[\mathrm{SD}])$} \\
\hline Visit 1 & $93.2(5.0)$ & $95.6(8.0)$ & $96.6(9.8)$ & $90.0(-)$ \\
\hline Visit 2 & $84.7(8.6)$ & $84.8(9.1)$ & $86.4(7.2)$ & $90.0(-)$ \\
\hline $\begin{array}{l}\text { Change from Visit } 1 \text { (mean } \\
\text { [95\% CI]) }\end{array}$ & $-8.5(-11.4,-5.7)$ & $-10.8(-14.0,-7.6)$ & $-10.3(-16.5,-4.1)$ & $0(-)$ \\
\hline$P$ value & $<0.001$ & $<0.001$ & 0.125 & - \\
\hline
\end{tabular}

Visit 2 readings were statistically compared against Visit 1 readings

$A H D$ antihypertensive drug, $C I$ confidence interval, $D B P$ diastolic blood pressure, $S B P$ systolic blood pressure, $S D$ standard deviation

supporting the effectiveness of amlodipine in a real-world scenario. Prescription of amlodipine either as monotherapy or add-on therapy reduced overall mean SBP and DBP by $13.6 \mathrm{mmHg}$ and $8.0 \mathrm{mmHg}$, respectively.
The majority of the patients in the present study (90.7\%) were prescribed amlodipine either as monotherapy or along with one other AHD. These findings are in line with those of earlier studies from different Indian cities [11, 13, 15] 
Table 4 Effect of amlodipine on blood pressure—subgroup analysis according to co-morbid condition and gender

\begin{tabular}{|c|c|c|c|c|}
\hline $\begin{array}{l}\text { Patients with hypertension } \\
\text { and diabetes }\end{array}$ & $\begin{array}{l}\text { Amlodipine monotherapy } \\
(n=67)\end{array}$ & $\begin{array}{l}\text { Amlodipine + } 1 \text { AHD } \\
(n=83)\end{array}$ & $\begin{array}{l}\text { Amlodipine + 2AHDs } \\
(n=20)\end{array}$ & $\begin{array}{l}\text { Amlodipine + 3AHDs } \\
(n=3)\end{array}$ \\
\hline \multicolumn{5}{|l|}{$\mathrm{SBP}(\mathrm{mmHg}$, mean $[\mathrm{SD}])$} \\
\hline Visit 1 & $161.6(16.4)$ & $163.0(14.8)$ & $169.6(22.9)$ & $170.0(21.6)$ \\
\hline Visit 2 & $147.4(21.4)$ & $145.2(19.9)$ & $155.7(30.9)$ & $156.7(9.4)$ \\
\hline $\begin{array}{l}\text { Change from Visit } 1 \text { (mean } \\
{[95 \% \mathrm{CI}] \text { ) }}\end{array}$ & $-14.1(-19.9,-8.4)$ & $-17.8(-22.5,-13.1)$ & $-13.9(-30.3,2.5)$ & $-13.3(-43.0,16.4)$ \\
\hline$P$ value & 0.002 & $<0.001$ & 1.0 & 1.0 \\
\hline \multicolumn{5}{|l|}{$\mathrm{DBP}(\mathrm{mmHg}$, mean $[\mathrm{SD}])$} \\
\hline Visit 1 & $95.5(6.8)$ & $95.6(7.9)$ & $96.0(9.0)$ & $98.0(2.8)$ \\
\hline Visit 2 & $87.4(10.6)$ & $85.6(9.4)$ & $90.5(11.0)$ & $86.7(4.7)$ \\
\hline $\begin{array}{l}\text { Change from Visit } 1 \text { (mean } \\
{[95 \% \mathrm{CI}] \text { ) }}\end{array}$ & $-8.0(-10.6,-5.4)$ & $-10.0(-12.5,-7.5)$ & $-5.5(-12.3,1.3)$ & $-11.3(-18.8,-3.8)$ \\
\hline$P$ value & $<0.001$ & $<0.001$ & 1.0 & 0.221 \\
\hline $\begin{array}{l}\text { Patients with hypertension } \\
\text { and dyslipidemia }\end{array}$ & $\begin{array}{l}\text { Amlodipine monotherapy } \\
(n=63)\end{array}$ & $\begin{array}{l}\text { Amlodipine + 1AHD } \\
(n=101)\end{array}$ & $\begin{array}{l}\text { Amlodipine + 2AHDs } \\
(n=20)\end{array}$ & $\begin{array}{l}\text { Amlodipine + 3AHDs } \\
(n=6)\end{array}$ \\
\hline \multicolumn{5}{|l|}{$\mathrm{SBP}(\mathrm{mmHg}$, mean $[\mathrm{SD}])$} \\
\hline Visit 1 & $158.7(13.7)$ & $162.5(17.2)$ & $171.1(22.5)$ & $159.5(14.3)$ \\
\hline Visit 2 & $146.7(19.7)$ & $144.1(17.8)$ & $146.4(24.9)$ & $155.0(17.1)$ \\
\hline $\begin{array}{l}\text { Change from Visit } 1 \text { (mean } \\
{[95 \% \mathrm{CI}] \text { ) }}\end{array}$ & $-12.0(-17.6,-6.4)$ & $-18.4(-22.7,-14.2)$ & $-24.7(-37.0,-12.4)$ & $-4.5(-12.0,3.0)$ \\
\hline$P$ value & 0.013 & $<0.001$ & 0.027 & 1.0 \\
\hline \multicolumn{5}{|l|}{$\mathrm{DBP}(\mathrm{mmHg}$, mean [SD]) } \\
\hline Visit 1 & $94.9(6.1)$ & $94.9(6.9)$ & $96.3(9.6)$ & $96.3(5.3)$ \\
\hline Visit 2 & $85.6(10.1)$ & $86.0(9.7)$ & $87.2(10.1)$ & $90.0(5.8)$ \\
\hline $\begin{array}{l}\text { Change from Visit } 1 \text { (mean } \\
{[95 \% \mathrm{CI}] \text { ) }}\end{array}$ & $-9.3(-12.2,-6.4)$ & $-8.9(-11.0,-6.8)$ & $-9.1(-15.5,-2.7)$ & $-6.3(-11.9,-0.70)$ \\
\hline$P$ value & $<0.001$ & $<0.001$ & 0.291 & 0.643 \\
\hline $\begin{array}{l}\text { Male patients with hyperten- } \\
\text { sion }\end{array}$ & $\begin{array}{l}\text { Amlodipine monotherapy } \\
(n=111)\end{array}$ & $\begin{array}{l}\text { Amlodipine + 1 AHD } \\
(n=84)\end{array}$ & $\begin{array}{l}\text { Amlodipine }+2 \text { AHDs } \\
(n=20)\end{array}$ & $\begin{array}{l}\text { Amlodipine + 3AHDs } \\
(n=5)\end{array}$ \\
\hline \multicolumn{5}{|l|}{$\mathrm{SBP}(\mathrm{mmHg}$, mean $[\mathrm{SD}])$} \\
\hline Visit 1 & $159.2(14.7)$ & $160.0(17.0)$ & $164.6(22.5)$ & $154.0(8.0)$ \\
\hline Visit 2 & $148.3(20.0)$ & $141.8(19.3)$ & $148.4(25.4)$ & $150.0(14.1)$ \\
\hline $\begin{array}{l}\text { Change from Visit } 1 \text { (mean } \\
{[95 \% \mathrm{CI}] \text { ) }}\end{array}$ & $-10.9(-14.7,-7.1)$ & $-18.1(-22.6,-13.6)$ & $-16.2(-30.0,-2.4)$ & $-4.0(-12.9,4.9)$ \\
\hline$P$ value & $<0.001$ & $<0.001$ & 0.575 & 1.0 \\
\hline \multicolumn{5}{|l|}{$\mathrm{DBP}(\mathrm{mmHg}$, mean [SD]) } \\
\hline Visit 1 & $96.3(6.8)$ & $95.6(7.0)$ & $96.5(9.6)$ & $94.8(4.5)$ \\
\hline Visit 2 & $88.9(11.0)$ & $86.0(11.0)$ & $88.7(11.1)$ & $88.0(4.0)$ \\
\hline $\begin{array}{l}\text { Change from Visit } 1 \text { (mean } \\
{[95 \% \mathrm{CI}] \text { ) }}\end{array}$ & $-7.4(-9.4,-5.4)$ & $-9.6(-11.6,-7.6)$ & $-7.8(-14.5,-1.1)$ & $-6.8(-13.4,-0.23)$ \\
\hline$P$ value & $<0.001$ & $<0.001$ & 0.589 & 0.776 \\
\hline $\begin{array}{l}\text { Female patients with hyper- } \\
\text { tension }\end{array}$ & $\begin{array}{l}\text { Amlodipine monotherapy } \\
(n=115)\end{array}$ & $\begin{array}{l}\text { Amlodipine + 1AHD } \\
(n=109)\end{array}$ & $\begin{array}{l}\text { Amlodipine + 2AHDs } \\
(n=16)\end{array}$ & $\begin{array}{l}\text { Amlodipine + 3AHDs } \\
(n=2)\end{array}$ \\
\hline \multicolumn{5}{|l|}{$\mathrm{SBP}(\mathrm{mmHg}$, mean $[\mathrm{SD}])$} \\
\hline Visit 1 & $160.7(14.7)$ & $164.5(14.9)$ & $166.9(16.5)$ & $193.5(6.5)$ \\
\hline Visit 2 & $147.4(20.0)$ & $146.9(20.0)$ & $156.4(28.4)$ & $165.0(15.0)$ \\
\hline $\begin{array}{l}\text { Change from Visit } 1 \text { (mean } \\
{[95 \% \mathrm{CI}] \text { ) }}\end{array}$ & $-13.3(-17.3,-9.3)$ & $-17.6(-22.0,-13.2)$ & $-10.5(-25.8,4.8)$ & $-28.5(-58.3,1.3)$ \\
\hline$P$ value & $<0.001$ & $<0.001$ & 1.0 & 0.889 \\
\hline $\mathrm{DBP}(\mathrm{mmHg}$, mean $[\mathrm{SD}])$ & & & & \\
\hline
\end{tabular}


Table 4 (continued)

\begin{tabular}{llllr}
\hline $\begin{array}{l}\text { Female patients with hyper- } \\
\text { tension }\end{array}$ & $\begin{array}{l}\text { Amlodipine monotherapy } \\
(n=115)\end{array}$ & $\begin{array}{l}\text { Amlodipine+1AHD } \\
(n=109)\end{array}$ & $\begin{array}{l}\text { Amlodipine +2AHDs } \\
(n=16)\end{array}$ & $\begin{array}{l}\text { Amlodipine + 3AHDs } \\
(n=2)\end{array}$ \\
\hline Visit 1 & $96.4(6.5)$ & $96.4(7.6)$ & $96.3(4.5)$ & $102.0(2.0)$ \\
Visit 2 & $88.8(9.7)$ & $87.0(9.4)$ & $90.6(9.2)$ & $90.0(10.0)$ \\
Change from Visit 1 (mean & $-7.6(-9.5,-5.7)$ & $-9.4(-11.6,-7.2)$ & $-5.6(-9.8,-1.4)$ & $-12.0(-23.1,-$ \\
$\quad[95 \%$ CI]) & & & $0.91)$ & 0.705 \\
$P$ value & $<0.001$ & $<0.001$ & 0.380 & \\
\hline
\end{tabular}

Visit 2 readings were statistically compared against Visit 1 readings

$A H D$ antihypertensive drug, $C I$ confidence interval, $D B P$ diastolic blood pressure, $S B P$ systolic blood pressure, $S D$ standard deviation

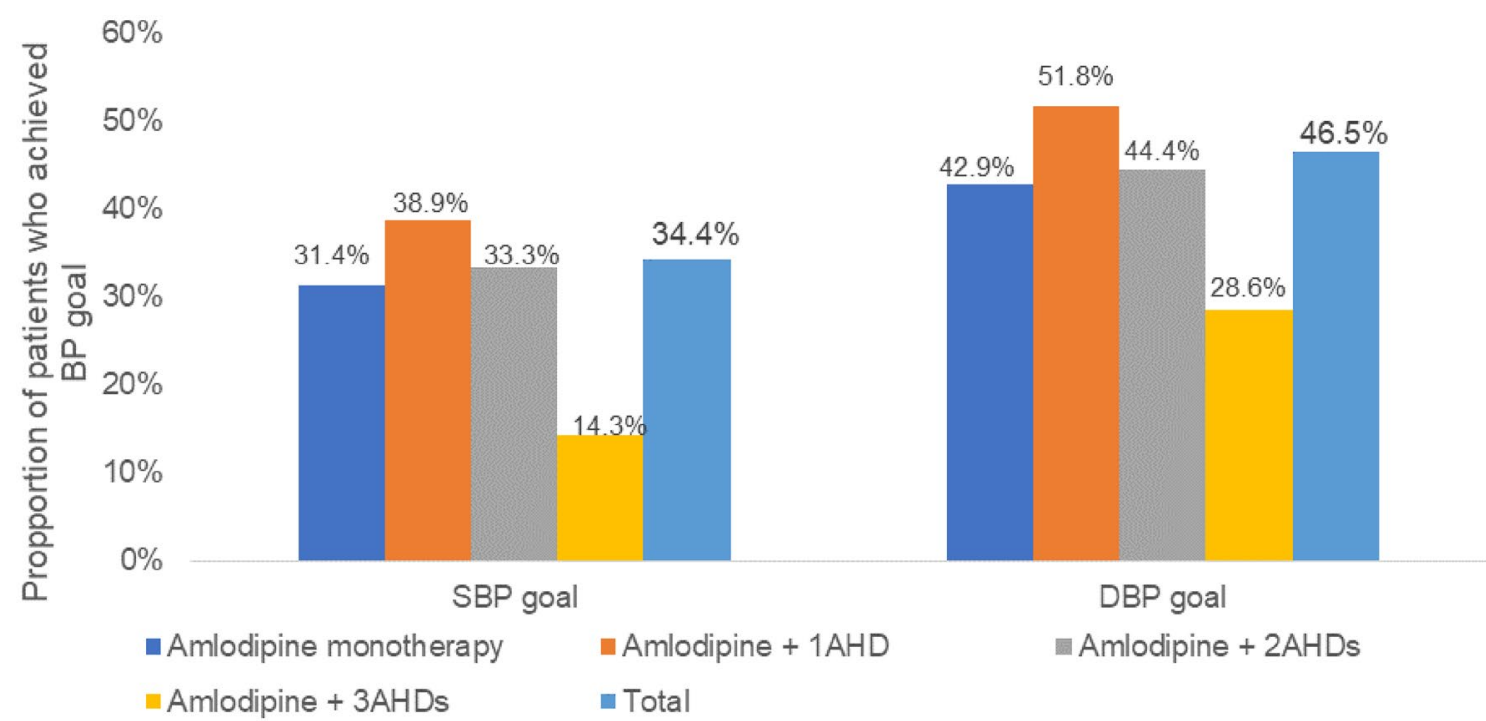

Fig. 3 Proportion of patients with essential hypertension who achieved individualized blood pressure goals after therapy with amlodipine as per ESC/ESH 2018 guidelines. SBP target was $<140 \mathrm{mmHg}$ and DBP target was $<90 \mathrm{mmHg}$. AHD antihyper-

that reported high prescription of CCBs, with amlodipine being the drug of choice for monotherapy or as combination therapy with other AHDs. The AHDs prescribed along with amlodipine in the present study cannot be stated as we did not analyze specific AHDs or classes that were prescribed along with amlodipine.

Reduction in $\mathrm{BP}$ across the amlodipine-treated groups was excellent-SBP: $11.0-17.8 \mathrm{mmHg}$ and DBP: $6.8-9.5 \mathrm{mmHg}$. Overall, $34.4 \%$ of the patients achieved SBP goal and $46.5 \%$ achieved DBP goal as specified in the ESC/ ESH 2018 and 2019 IGH-IV guidelines $(<140 / 90 \mathrm{mmHg})$. These findings are in line with those of previous EMR-based studies from the USA wherein amlodipine was prescribed as monotherapy or in combination with other AHDs [29-31]. These studies were conducted over different time periods: 1998-2001 [29], 1998-2005 [30], and 2005-2010 [31]. Here, mean reduction in SBP from baseline was $17.2 \mathrm{mmHg}$, tensive drug, $B P$ blood pressure, DBP diastolic BP, ESC/ESH European Society of Cardiology/European Society of Hypertension, $S B P$ systolic BP

$13.3 \mathrm{mmHg}$, and $18.2 \mathrm{mmHg}$, while mean reduction in DBP was $8.3 \mathrm{mmHg}, 6.1 \mathrm{mmHg}$, and $8.4 \mathrm{mmHg}$, in the three studies, respectively. The mean proportion of patients who achieved the SBP and DBP goals $(<140 / 90 \mathrm{mmHg})$ was $42.4 \%, 46.4 \%$, and $44.2 \%$ in the three studies, respectively.

A reduction in BP was observed across age groups in the current study. In patients aged $\leq 45$ years, a reduction of 10.0-27.1 mmHg in SBP and 7.2-10.6 mmHg in DBP was observed, with $39.8 \%$ and $40.8 \%$ of the patients achieving the SBP and DBP goals specified in the ESC/ESH 2018 and 2019 IGH-IV guidelines $(<140 / 90 \mathrm{mmHg}$ ). However, similar studies conducted in this age group are not available for comparison. In elderly patients (age $\geq 65$ years) a reduction of 13.9-22.4 mmHg in SBP and $8.5-10.8 \mathrm{mmHg}$ in DBP was observed, with $27.2 \%$ of the patients achieving SBP goal and merely $12.0 \%$ achieving the DBP goal specified in the ESC/ESH 2018 and 2019 IGH-IV guidelines 


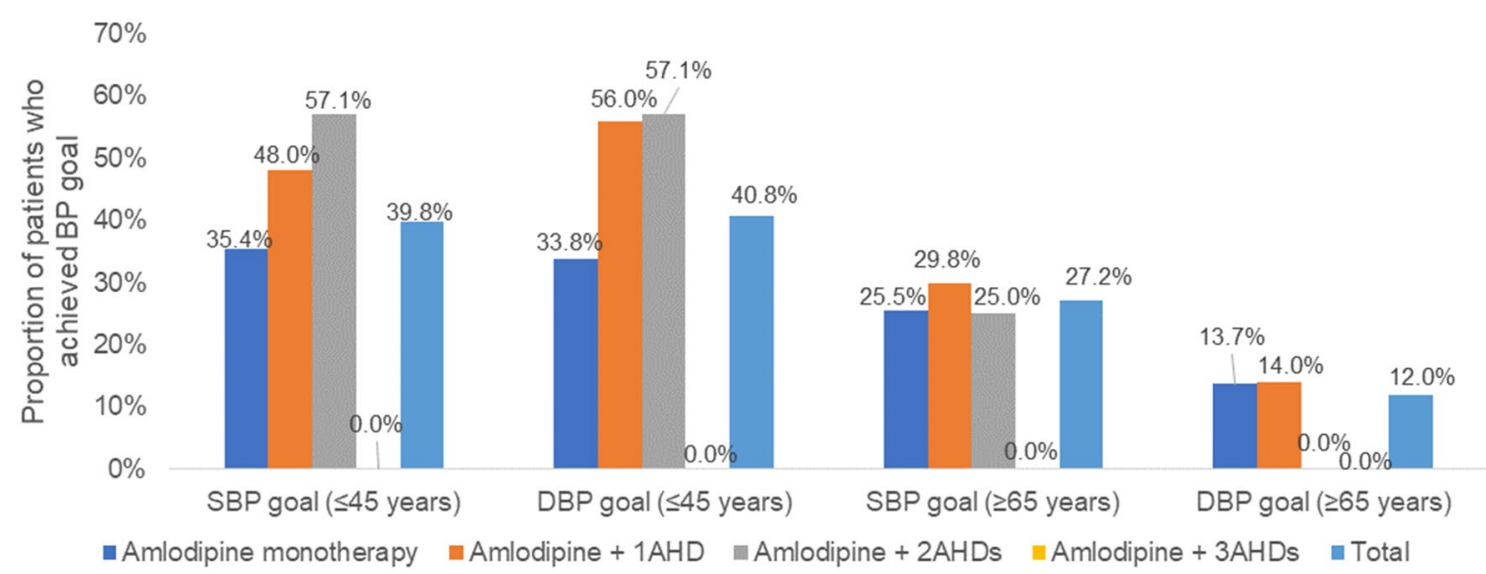

Fig. 4 Proportion of patients with essential hypertension who achieved individualized blood pressure goals after therapy with amlodipine as per ESC/ESH 2018 guidelines-subgroup analysis by age. SBP target was $<140 \mathrm{mmHg}$ and DBP target was $<90 \mathrm{mmHg}$ for patients aged $\leq 45$ years. SBP target was $<140 \mathrm{mmHg}$ and DBP target was $<80 \mathrm{mmHg}$ for patients aged $\geq 65$ years. $A H D$ antihypertensive drug, $B P$ blood pressure, DBP diastolic BP, ESC/ESH European Society of Cardiology/European Society of Hypertension, $S B P$ systolic BP

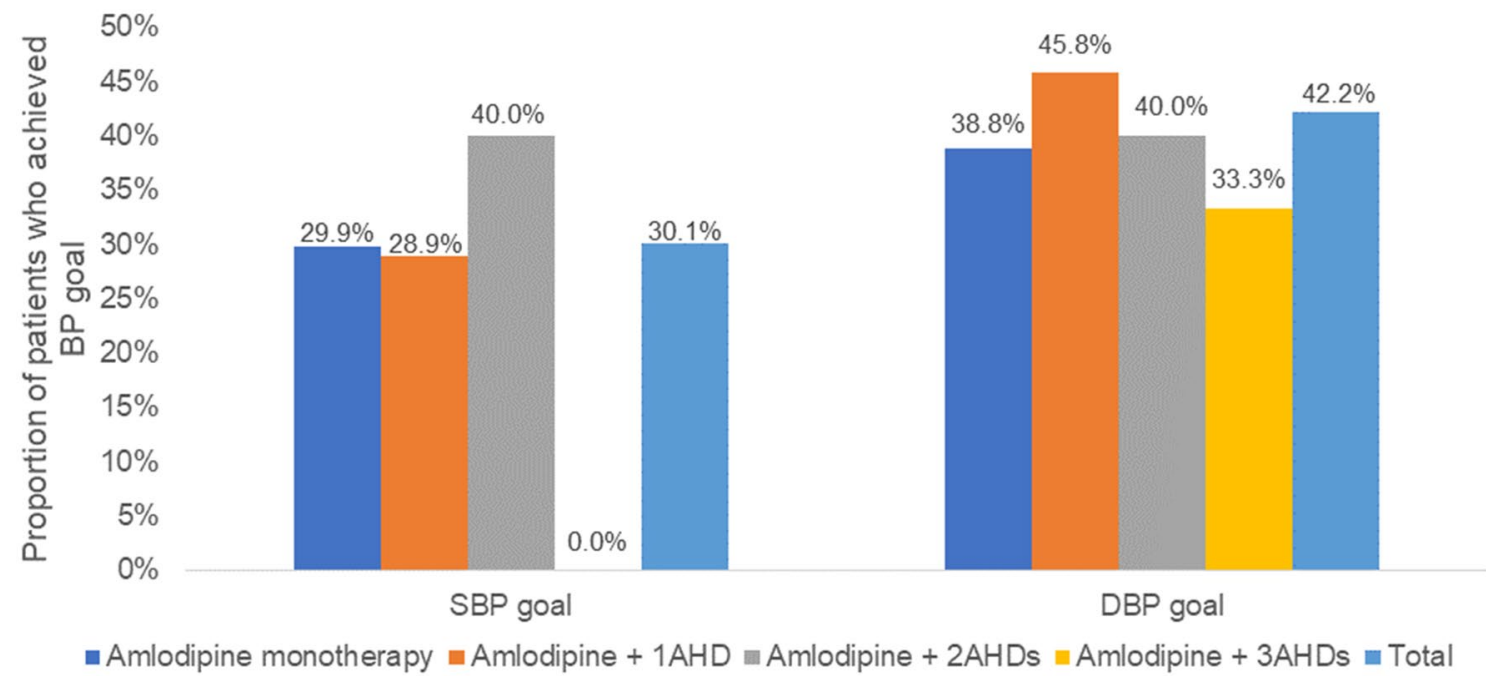

Fig. 5 Proportion of patients with essential hypertension and diabetes who achieved individualized blood pressure goals after therapy with amlodipine as per ESC/ESH 2018 guidelines. SBP target was $\leq 130 \mathrm{mmHg}$ and DBP target was $\leq 80 \mathrm{mmHg}$. AHD antihyper-

$(<140 / 80 \mathrm{mmHg})$. The relatively low proportion of elderly patients achieving BP goals could be due to the small number of people included in the study. The reduction in BP was similar to that reported by Bisognano et al. (mean reduction in SBP $15.8 \mathrm{mmHg}$ ) [29] and Weycker et al. (reduction in SBP $14.9 \mathrm{mmHg}$ and DBP $6.4 \mathrm{mmHg}$ ) [30].

In the current study, amlodipine-based regimens reduced SBP (13.3-17.8 $\mathrm{mmHg})$ and DBP $(5.5-11.3 \mathrm{mmHg})$ in patients with essential hypertension and co-morbid diabetes; similar to earlier studies that reported a mean reduction of $14.6 \mathrm{mmHg}, 15.3 \mathrm{mmHg}$, and $17.7 \mathrm{mmHg}$ in $\mathrm{SBP}$ tensive drug, BP blood pressure, DBP diastolic BP, ESC/ESH European Society of Cardiology/European Society of Hypertension, SBP systolic BP

and $5.3 \mathrm{mmHg}$ and $9.1 \mathrm{mmHg}$ in DBP [29-31]. The ESC/ ESH 2018 recommended BP target $(\leq 130 / 80 \mathrm{mmHg})$ was achieved by $30.1 \%$ of patients for SBP and $42.2 \%$ for DBP in the current study. These results were higher than the proportion of patients who achieved the same goal as reported by Bisognano et al. (16.3\%) [29] and Ram et al. (21.1\%) [31], but were comparable with Weycker et al.'s findings (45.9\%) [30].

In India, CCBs such as amlodipine have been reported to be preferred as an AHD for elderly patients [32, 33]. This approach is in line with clinical trials conducted in 


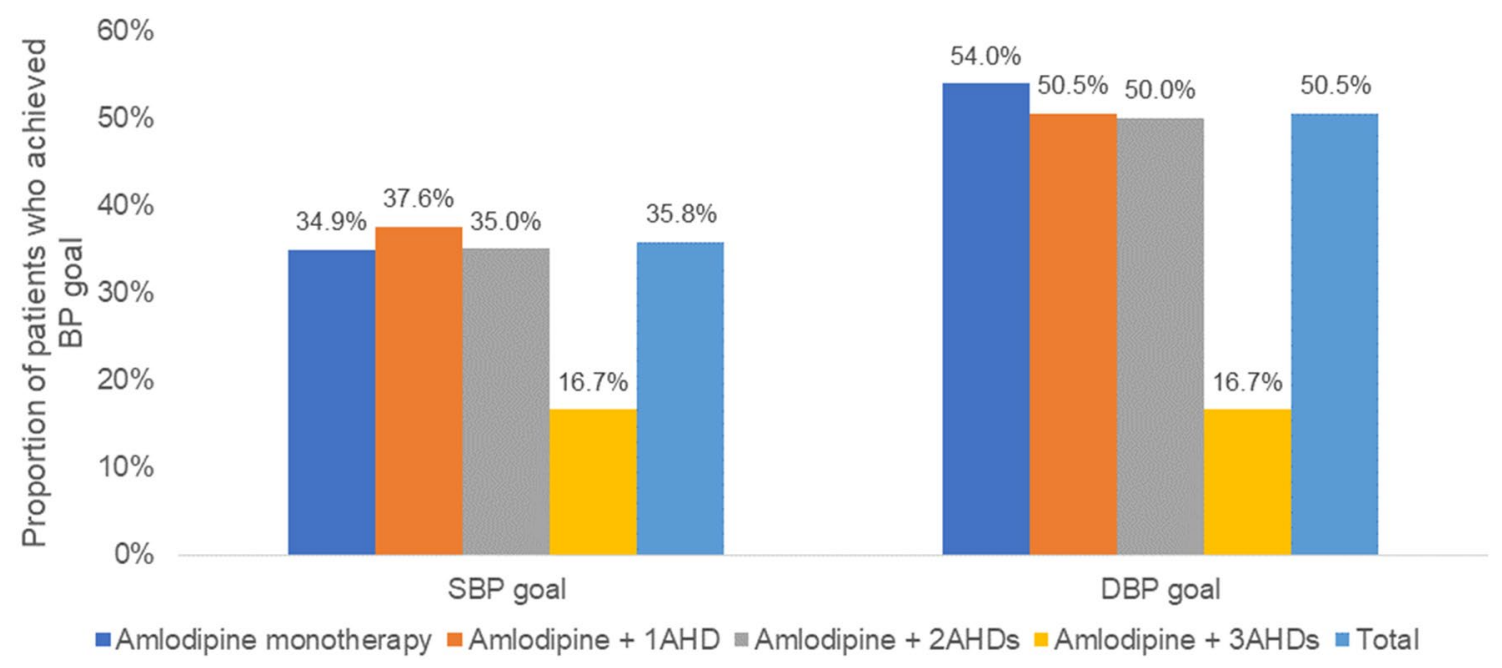

Fig. 6 Proportion of patients with essential hypertension and dyslipidemia who achieved individualized blood pressure goals after therapy with amlodipine. SBP target was $<140 \mathrm{mmHg}$ and DBP tar-

young and elderly patients that report a greater response to amlodipine among the elderly compared to younger patients [34-36], probably due to a higher plasma concentration in the elderly [35]. However, Ramakrishnan et al. [2] recently reported that the prevalence of hypertension in Indian adults aged $<45$ years is $34.6 \%$. This is worrying because hypertension is a major risk factor for other cardiovascular diseases [37] and puts young Indians at an increased risk of premature mortality due to cardiovascular disorders [38]. The current study reports that amlodipine as monotherapy or add-on therapy with other AHDs is effective in reducing blood pressure in individuals aged $\leq 45$ years and the elderly, even in the presence of co-morbidities. Hence, clinicians may consider prescribing amlodipine to individuals across age groups and co-morbid conditions.

\subsection{Limitations}

The retrospective design of this study is a major limitation. Moreover, unlike clinical trials where supervised drug administration takes place, patients in the current study were responsible for adhering to and complying with the given prescription. Due to the stringent inclusion criteria, the sample size in a few subgroups was quite small. However, future studies can be performed with a larger sample size to validate the findings obtained in the current study. As EMR records only contain prescription data, we cannot exclude the possibility that some patients may not have adhered to the prescription, resulting in one or multiple missed doses, which could have led to a low observed therapeutic effect. As the objective of the study was to evaluate the effectiveness of amlodipine and not safety or tolerability, we did not evaluate any safety or tolerability outcomes. Also, the EMR get was $<90 \mathrm{mmHg}$. $A H D$ antihypertensive drug, $B P$ blood pressure, $D B P$ diastolic BP, $S B P$ systolic BP

database does not capture adverse events or clinical outcomes such as mortality, hospitalization, and cardiovascular outcomes. BP was measured at various centers using different methods instead of a uniform protocol, which may have caused a variation in the measurements. In the combination groups (i.e., amlodipine + other AHDs), usage of different AHDs may have different effect on BP reduction. However, we did not perform matching across these groups to adjust for this effect.

\section{Conclusion}

The current study is one of the first EMR-based studies in India to assess the effectiveness of amlodipine, used either as monotherapy or in combination with other AHDs, in reducing SBP and DBP in Indian patients with mild to moderate essential hypertension. We observed that amlodipine prescribed as monotherapy or add-on therapy during routine clinical practice significantly reduced blood pressure in $\leq 45$ and $\geq 65$-year-old Indian patients with mild to moderate hypertension. These findings emphasize that amlodipine may be a good candidate for blood pressure control in Indian patients with essential hypertension.

Acknowledgements The authors thank Leo J. Philip Tharappel (SIRO Clinpharm Pvt Ltd.) for providing medical writing assistance.

\section{Declarations}

Funding This study was funded by Dr. Reddy's Laboratories.

Conflict of interest MYK, SP, AM, and SM are employees of Dr. Reddy's Laboratories and may own stock. SS is an employee of Healthplix 
Ltd, which received consultancy fees from Dr. Reddy's Laboratories to perform the study. SR, JCM, BCS, and SRK are members of the advisory board for Dr. Reddy's Laboratories.

Ethics approval The study protocol was approved by an independent ethics committee on 3 December 2019.

Consent to participate Not applicable.

Consent for publication Not applicable.

Availability of data and material The datasets generated during and/or analyzed during the current study are available from the corresponding author on reasonable request.

Code availability Not applicable.

Author contributions MYK, SP, AM, SM, and SS developed the concept and performed the study. SS performed data analysis. MYK and SP drafted the manuscript. All authors reviewed the manuscript and gave final approval.

Open Access This article is licensed under a Creative Commons Attribution-NonCommercial 4.0 International License, which permits any non-commercial use, sharing, adaptation, distribution and reproduction in any medium or format, as long as you give appropriate credit to the original author(s) and the source, provide a link to the Creative Commons licence, and indicate if changes were made. The images or other third party material in this article are included in the article's Creative Commons licence, unless indicated otherwise in a credit line to the material. If material is not included in the article's Creative Commons licence and your intended use is not permitted by statutory regulation or exceeds the permitted use, you will need to obtain permission directly from the copyright holder. To view a copy of this licence, visit http://creativecommons.org/licenses/by-nc/4.0/.

\section{References}

1. Collaboration NCDRF. Worldwide trends in blood pressure from 1975 to 2015: a pooled analysis of 1479 population-based measurement studies with 19.1 million participants. Lancet. 2017;389(10064):37-55. https://doi.org/10.1016/s0140 $-6736(16) 31919-5$.

2. Ramakrishnan S, Zachariah G, Gupta K, Shivkumar Rao J, Mohanan PP, Venugopal K, et al. Prevalence of hypertension among Indian adults: results from the great India blood pressure survey. Indian Heart J. 2019;71(4):309-13. https://doi. org/10.1016/j.ihj.2019.09.012.

3. Global Burden of Disease Collaborative Network. Global Burden of Disease Study 2017 (GBD 2017) Results. Seattle, United States: Institute for Health Metrics and Evaluation (IHME); 2018.

4. Paramore LC, Halpern MT, Lapuerta P, Hurley JS, Frost FJ, Fairchild DG, et al. Impact of poorly controlled hypertension on healthcare resource utilization and cost. Am J Manag Care. 2001;7(4):389-98.

5. Anchala R, Kannuri NK, Pant H, Khan H, Franco OH, Di Angelantonio E, et al. Hypertension in India: a systematic review and meta-analysis of prevalence, awareness, and control of hypertension. J Hypertens. 2014;32(6):1170-7. https://doi.org/10.1097/ HJH.0000000000000146.

6. Prenissl J, Manne-Goehler J, Jaacks LM, Prabhakaran D, Awasthi A, Bischops AC, et al. Hypertension screening, awareness, treatment, and control in India: a nationally representative cross-sectional study among individuals aged 15 to 49 years. PLoS Med. 2019;16(5):e1002801. https://doi.org/10.1371/journ al.pmed.1002801.

7. James PA, Oparil S, Carter BL, Cushman WC, Dennison-Himmelfarb C, Handler J, et al. 2014 evidence-based guideline for the management of high blood pressure in adults: report from the panel members appointed to the Eighth Joint National Committee (JNC 8). JAMA. 2014;311(5):507-20. https://doi.org/10.1001/ jama.2013.284427.

8. Whelton PK, Carey RM, Aronow WS, Casey DE Jr, Collins KJ, Dennison Himmelfarb C, et al. 2017 ACC/AHA/AAPA/ ABC/ACPM/AGS/APhA/ASH/ASPC/NMA/PCNA guideline for the prevention, detection, evaluation, and management of high blood pressure in adults: executive summary: A Report of the American College of Cardiology/American Heart Association Task Force on Clinical Practice Guidelines. Hypertension. 2018;71(6):1269-324. https://doi.org/10.1161/HYP.00000 00000000066 .

9. Williams B, Mancia G, Spiering W, Agabiti Rosei E, Azizi M, Burnier M, et al. 2018 ESC/ESH Guidelines for the management of arterial hypertension. Eur Heart J. 2018;39(33):3021-104. https ://doi.org/10.1093/eurheartj/ehy339.

10. AoPo India. Indian guidelines on hypertension-IV 2019. J Assoc Physicians India. 2019;67(suppl 2):8-46.

11. Dasbiswas A, Tamboli T, Ingole S, Naik S, Jain R. Management of hypertension- Insights into real-world clinical practice for differential usage of calcium channel blockers (CCBS). J Evid Based Med Healthc. 2017;4(38):2295-9. https://doi.org/10.18410/jebmh /2017/451.

12. Ramakrishnan S, Ingole S, Dey A, Jain R. Management of hypertension: insights into prescribing behavior with focus on angiotensin receptor blockers. J Pract Cardiovasc Sci. 2017;3(1):22-7.

13. Tiwari H, Kumar A, Kulkarni SK. Prescription monitoring of anti-hypertensive drug utilisation at the Panjab University Health Centre in India. Singapore Med J. 2004;45(3):117-20.

14. Varakantham V, Kurakula Sailoo AK, Bharatraj DK. Antihypertensive prescription pattern and compliance to JNC 7 and JNC 8 at tertiary care government hospital, Hyderabad, India: a crosssectional retrospective study. Hosp Pharm. 2018;53(2):107-12. https://doi.org/10.1177/0018578717738080.

15. Sreedharan N, Rao PG, Rau NR, Shankar PR. Antihypertensive prescribing preferences in three South Indian Hospitals: cost analysis, physicians perspectives and emerging trends. Int J Clin Pharmacol Ther. 2011;49(4):277-85. https://doi.org/10.5414/ CP201464.

16. Mankadavath A, Chandrasekhar D, Thomas T, Zuhra F, Kaipanthodi S, Parambil JC. A prospective drug use evaluation of antihypertensive drugs in in-patients of a tertiary referral care hospital. J Basic Clin Physiol Pharmacol. 2015;26(3):295-300. https://doi. org/10.1515/jbcpp-2013-0131.

17. Das AK, Chatterjee S, Pal J. Clinical effectiveness and safety of low cost versus innovator brand amlodipine in hypertension: a single-blinded, randomized, crossover, noninferiority trial. Indian J Pharmacol. 2016;48(6):706-9. https://doi.org/10.4103/02537613.194844.

18. Devi P, Xavier D, Sigamani A, Pandey S, Thomas T, Murthy $S$, et al. Effect of fixed dose combinations of metoprolol and amlodipine in essential hypertension: MARS - a randomized controlled trial. Blood Press Suppl. 2011;2:5-12. https://doi. org/10.3109/08037051.2011.617040.

19. Hiremath JS, Chokalingam K, Mathan G, Reddy PNC, Sharma A, Dhawan S, et al. A randomized, double-blinded, controlled, multicentre phase III study to evaluate the efficacy and safety of telmisartan/amlodipine/hydrochlorothiazide compared to 
telmisartan/hydrochlorothiazide in patients with essential hypertension. J Assoc Physicians India. 2018;66(12):11-2.

20. Pareek A, Salkar H, Mulay P, Desai S, Chandurkar N, Redkar N. A randomized, comparative, multicentric evaluation of atenolol/amlodipine combination with atenolol alone in essential hypertensive patients. Am J Ther. 2010;17(1):46-52. https://doi. org/10.1097/MJT.0b013e3181a9db74.

21. Bahl VK, Jadhav UM, Thacker HP. Management of hypertension with the fixed combination of perindopril and amlodipine in daily clinical practice: results from the STRONG prospective, observational, multicenter study. Am J Cardiovasc Drugs. 2009;9(3):135-42. https://doi.org/10.2165/00129784-20090903000001 (10.1007/bf03256570).

22. Altman DG, Bland JM. How to obtain the $P$ value from a confidence interval. BMJ. 2011;343:d2304. https://doi.org/10.1136/ bmj.d2304.

23. Palma-Gamiz JL. High blood pressure and calcium antagonism. Cardiology. 1997;88(Suppl 1):39-46. https://doi. org/10.1159/000177461.

24. Fares H, DiNicolantonio JJ, O'Keefe JH, Lavie CJ. Amlodipine in hypertension: a first-line agent with efficacy for improving blood pressure and patient outcomes. Open Heart. 2016;3(2):e000473. https://doi.org/10.1136/openhrt-2016-000473.

25. Sison J, Assaad-Khalil SH, Najem R, Kitchlew AR, Cho B, Ueng $\mathrm{KC}$, et al. Real-world clinical experience of amlodipine/valsartan and amlodipine/valsartan/hydrochlorothiazide in hypertension: the EXCITE study. Curr Med Res Opin. 2014;30(10):1937-45. https://doi.org/10.1185/03007995.2014.942415.

26. Hu D, Liu L, Li W. Efficacy and safety of valsartan/amlodipine single-pill combination in 11,422 Chinese patients with hypertension: an observational study. Adv Ther. 2014;31(7):762-75. https ://doi.org/10.1007/s12325-014-0132-x.

27. Khan W, Moin N, Iktidar S, Sakrani J, Abid R, Afzal J, et al. Reallife effectiveness, safety, and tolerability of amlodipine/valsartan or amlodipine/valsartan/hydrochlorothiazide single-pill combination in patients with hypertension from Pakistan. Ther Adv Cardiovasc Dis. 2014;8(2):45-55. https://doi.org/10.1177/17539 44714525496.

28. Lee SA, Choi HM, Park HJ, Ko SK, Lee HY. Amlodipine and cardiovascular outcomes in hypertensive patients: meta-analysis comparing amlodipine-based versus other antihypertensive therapy. Korean J Intern Med. 2014;29(3):315-24. https://doi. org/10.3904/kjim.2014.29.3.315.

29. Bisognano J, McLaughlin T, Roberts CS, Battleman D, Schwartz B, Garza D, et al. Incremental effectiveness of amlodipine besylate in the treatment of hypertension with single and multiple medication regimens. Am J Hypertens. 2004;17(8):676-83. https://doi. org/10.1016/j.amjhyper.2004.04.007.

30. Weycker D, Keskinaslan A, Levy DG, Edelsberg J, Kartashov A, Oster G. Effectiveness of add-on therapy with amlodipine in hypertensive patients receiving valsartan. Blood Press Suppl. 2008;2:5-12. https://doi.org/10.1080/08038020802316536.

31. Ram CV, Vasey J, Panjabi S, Qian C, Quah R. Comparative effectiveness analysis of amlodipine/renin-angiotensin system blocker combinations. J Clin Hypertens (Greenwich). 2012;14(9):601-10. https://doi.org/10.1111/j.1751-7176.2012.00695.x.

32. Gupta SK, Nayak RP. The pattern of antihypertensive medication use among elderly patients in a tertiary care teaching hospital in South India. Trop J Med Res. 2014;17(2):81-5.

33. Mohd AH, Mateti UV, Konuru V, Parmar MY, Kunduru BR. A study on prescribing patterns of antihypertensives in geriatric patients. Perspect Clin Res. 2012;3(4):139-42.

34. Abernethy DR, Gutkowska J, Lambert MD. Amlodipine in elderly hypertensive patients: pharmacokinetics and pharmacodynamics. J Cardiovasc Pharmacol. 1988;12(Suppl 7):S67-71. https://doi. org/10.1097/00005344-198812007-00015.

35. Abernethy DR, Gutkowska J, Winterbottom LM. Effects of amlodipine, a long-acting dihydropyridine calcium antagonist in aging hypertension: pharmacodynamics in relation to disposition. Clin Pharmacol Ther. 1990;48(1):76-86. https://doi.org/10.1038/ clpt.1990.120.

36. Kloner RA, Sowers JR, DiBona GF, Gaffney M, Wein M. Sexand age-related antihypertensive effects of amlodipine. The Amlodipine Cardiovascular Community Trial Study Group. Am J Cardiol. 1996;77(9):713-22. https://doi.org/10.1016/s0002 -9149(97)89205-3.

37. Kjeldsen SE. Hypertension and cardiovascular risk: general aspects. Pharmacol Res. 2018;129:95-9. https://doi.org/10.1016/j. phrs.2017.11.003.

38. Gupta R, Xavier D. Hypertension: the most important non communicable disease risk factor in India. Indian Heart J. 2018;70(4):565-72. https://doi.org/10.1016/j.ihj.2018.02.003.

\section{Affiliations}

\section{Mohammed Yunus Khan ${ }^{1}$. Sucheta Pandit ${ }^{1}$. Saumitra Ray ${ }^{2}$. Jagdish Chander Mohan ${ }^{3}$ - B. C. Srinivas ${ }^{4}$. Santosh Ramakrishnan ${ }^{5}$. Amey Mane ${ }^{1} \cdot$ Suyog Mehta ${ }^{1} \cdot$ Snehal Shah $^{6}$}

1 Dr. Reddy's Laboratories Ltd., 7-1-27, Ameerpet, Hyderabad, Telangana 500016, India

2 Vivekananda Institute of Medical Sciences, Kolkata, West Bengal, India

3 Fortis Hospital, Shalimar Bagh, Delhi, India
4 Sri Jayadeva Institute of Cardiology, Bengaluru, Karnataka, India

5 Magna Centres for Obesity Diabetes and Endocrinology, BTM 2nd Stage, Bengaluru, Karnataka, India

6 Healthplix Ltd., Bengaluru, Karnataka, India 